\title{
Achieving Phase and Amplitude Stability in Pulsed Superconducting Cavities
}

\author{
S. N. Simrock, DESY, Hamburg, Germany
}

\begin{abstract}
Superconducting cavities exhibit a high susceptibility to mechanical perturbations due to the narrow bandwidth of the cavities. Significant phase and amplitude errors can be induced by the frequency variations driven by microphonics and Lorentz force detuning especially in the case of pulsed operation where mechanical resonances of the cavity can be excited. While the relativistic electron and positron beams in the TESLA linacs will permit the control of the vector-sum of many cavities driven by one klystron, the non-relativistic proton beam in the linacs for SNS and the JAERI/KEK Joint Project limits the number of cavities that can be controlled by one klystron. Considerable experience of RF control at high gradients ( $>15 \mathrm{MV} /$ $\mathrm{m}$ ) with pulsed RF and pulsed beam has been gained at the TESLA Test Facility in which presently 16 cavities are driven by one klystron. The RF control system employs a completely digital feedback system to provide flexibility in the control algorithms, precise calibration of the vectorsum, and extensive diagnostics and exception handling capabilities. Presently under study is a piezotranslator based active compensation scheme for the time varying Lorentz force detuning which if successful will reduce RF power requirements at gradients $>25 \mathrm{MV} / \mathrm{m}$ considerably and provide improved field stability.
\end{abstract}

\section{INTRODUCTION}

Superconducting cavity technology has developed very rapidly over the past 15 years. While the first large scale applications at Argonne (ATLAS), JLAB (CEBAF), CERN (LEP), KEK (TRISTAN) are now in operation for many years, they have been limited to $\mathrm{CW}$ operation and relativistic electron beams.

Meanwhile significant progress has been made on the issues of pulsed operation of superconducting cavities at high gradients with respect to Lorentz force detuning and the associated resonant excitation of mechanical resonance, microphonics, the transients induced by the pulsed beam, and the control of the vector-sum of many cavities driven by one klystron. Based on the positive experience at the TESLA Test Facility where 16 cavities driven by one klystron are in operation since 1998, several new H- accelerator projects under construction or in planning have adopted superconducting cavities in their design. The challenge here is the non-relativistic nature of the beam which results in phase slippage along the linac and therefore imposes tight requirements on the stability of individual cavity fields. Furthermore the synchrotron oscillations induce changes in beam loading in the individual cavities. The parameters of the various pulsed superconducting accelerator are shown in Table 1.

Table 1: Pulsed Superconducting Accelerators under Construction or in Planning

\begin{tabular}{|c|c|c|c|c|c|}
\hline Accelerator & \multirow{2}{*}{ TESLA/TTF } & \multirow{2}{*}{ SNS } & \multirow{2}{*}{$\begin{array}{l}\text { Joint Project } \\
\text { JAERI/KEK }\end{array}$} & \multirow{2}{*}{ CONCERT } & \multirow{2}{*}{ SPL } \\
\hline Parameter & & & & & \\
\hline frequency [MHz] & 1300 & 805 & 972 & 704 & 352 \\
\hline gradient $[\mathrm{MV} / \mathrm{m}]$ & $23.4(35) / 15$ & $10.2 /$ & $9.9 / 10.5$ & $10.3 / 12.4$ & $3.5 / 5 / 9 / 7.5$ \\
\hline cavity beta & 1 & $0.61 / 0.81$ & $0.73 / 0.77$ & $0.68 / 0.86$ & $0.52 / 0.7 / 0.8 / 1$ \\
\hline number of cavities / klystron & $36 / 32$ & 1 & 2 & 1 & $1 / 1 / 4 / 6$ \\
\hline rf /beam pulse duration [ms] & $1.4 / 0.95$ & $1.17 / 1.04$ & $<1.0 / 0.5$ & $1.13 / 1.0$ & $<4.2 / 2.2$ \\
\hline repetition rate $[\mathrm{Hz}]$ & $5 / 10$ & 60 & 50 & 50 & 75 \\
\hline av. beam current in macro pulse $[\mathrm{mA}]$ & $9.5 / 8.0$ & 27.7 & 28 & 75 & 11 \\
\hline chopper on/off time [n/ns] or $\%$ & $\mathrm{n} / \mathrm{a}$ & $546 / 295(68 \%)$ & $54 \%$ & $360 / 240$ & $40 \%$ \\
\hline beam energy spread at linac end [\%] & 0.01 & 0.2 & $0.2(0.1)$ & 0.1 & 0.2 \\
\hline phase/ampl. stab. requ. [+-deg./+-\%] & $0.5 / 0.02(\mathrm{rms})$ & $0.5 / 0.5$ & $1.0 / 1.0$ & $0.5 / 0.5$ & $0.5 / 0.5$ \\
\hline loaded Q [1e6] & $3 / 3$ & $0.73 / 0.70$ & $\sim 0.7$ & $0.43 / 0.38$ & $2 / 2.5 / 3 / 2$ \\
\hline cavity bandwidth HWHM [Hz] & 220 & $550 / 500$ & $\sim 600$ & $1600 / 1800$ & $\sim 60$ \\
\hline expected microphonics [Hz] (rms) & $3-7$ & $100(6 \sigma)$ & 20 & 100 & 20 \\
\hline Lor. force det. const. $\left[\mathrm{Hz} /(\mathrm{MV} / \mathrm{m})^{\wedge} 2\right]$ & 1 & $2.9 / 0.7$ & $1.6 / 1.4$ & $8 / 4^{\mathrm{a}}$ & $0.75^{\mathrm{a}}(\beta=1)$ \\
\hline frequency (Q) of dominant mech. mode & $280(20)$ & & $122(60)^{b}$ & $40-60(100)$ & $\sim 100(\sim 40)^{\mathrm{a}}$ \\
\hline
\end{tabular}

a. assumption made for simulation

b. measured at $600 \mathrm{MHz}$ 


\section{RF CONTROL REQUIREMENTS}

The requirements for the RF control system are usually defined in terms of phase and amplitude stability of the accelerating field during the flat-top portion of the rf pulse which is shown in Figure 1. In addition operational demands may impose further needs on the design of the RF control system.

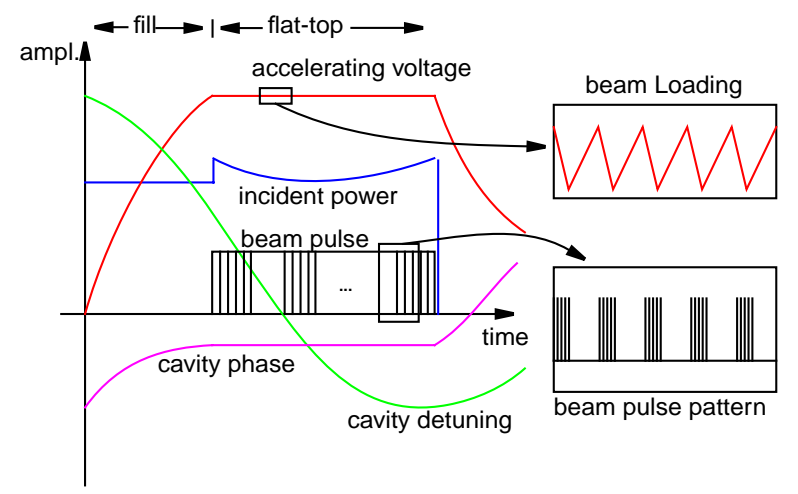

Fig. 1: Various rf system parameters for a pulsed superconducting cavity.

\section{Amplitude and Phase Stability}

The requirements for the RF control system are derived from the desired beam parameters such as bunch-to-bunch and pulse-to-pulse energy spread. In general it is desirable to maintain a constant momentum and time-of-arrival profile for the particle bunches along the linac to ensure well defined longitudinal and transverse beam characteristics.

The beam parameters can be translated into the requirements for phase and amplitude of the accelerating field of individual cavities or the vector-sum of several cavities driven by one klystron. While the TESLA collider requires tight field control of the order of $0.02 \%$ for the amplitude and $0.5 \mathrm{deg}$. for the phase, the requirements for the $\mathrm{H}$ linacs are relaxed to $0.5 \%$ and $0.5 \mathrm{deg}$. which are similar for all $\mathrm{H}^{-}$linacs. If the vector-sum is controlled, additional requirements are imposed on the accuracy of the calibration of the vector-sum which must be of the order of $10 \%$ for amplitude and 1\% for phase in presence of $+-10 \%$ microphonics in the case of TESLA.

\section{Operational Requirements}

The RF control system must be operable, reliable, reproducible, well understood and meet technical performance goals. Besides field stabilization the RF control system must provide diagnostics for the calibration of gradient and beam phase, measurement of the loop phase, cavity detuning, and control of the cavity frequency tuners. Exception handling capability must be implemented to avoid unnecessary beam loss. Features such as automated fault recovery will help to maximize accelerator up-time. A thorough understanding of the RF system will allow for operation close to the performance envelope while maximizing accelerator availability. Often the RF control must be fully functional over a wide range of operating parameters such as gradients and beam current. For efficiency reasons the RF system should provide sufficient control close to klystron saturation.

\section{SOURCES OF PERTURBATIONS}

The major sources of perturbations which have to be controlled by the low level RF system are fluctuations of the resonance frequency of the cavities and fluctuations of the beam current. Changes in resonance frequency result from deformations of the cavity walls induced by mechanical vibrations (microphonics) or the gradient dependent Lorentz force.

\section{Lorentz Force}

The static detuning of a resonator due to Lorentz force is proportional to the square of the accelerating field. In the case of pulsed RF fields the mechanical resonances will be excited resulting in a time varying cavity detuning even during the flat-top portion of the RF pulse where the gradient is constant. The lower the mechanical quality factor and the higher the mechanical resonance frequency (only longitudinal modes should be excited), the less likely is the enhancement of the peak cavity detuning by the Lorentz force. Stiffening rings at the iris are used to reduce the Lorentz force detuning constant and increase the mechanical resonance frequencies.

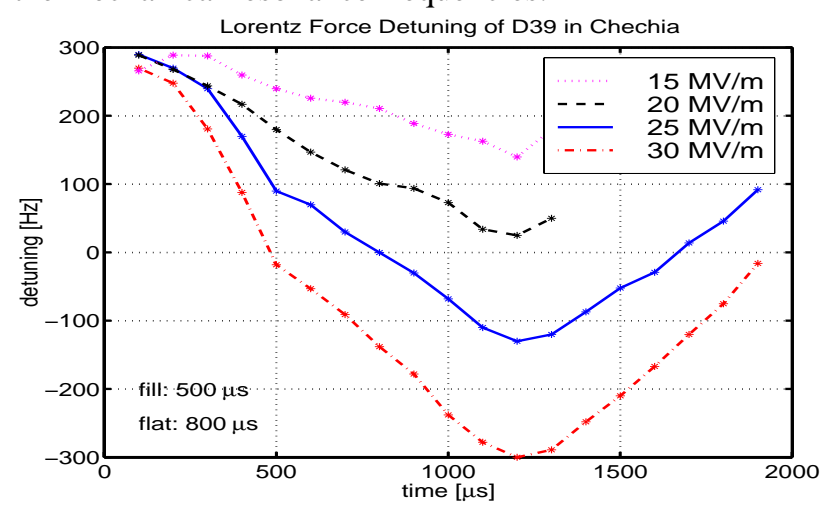

Fig. 2: Lorentz force detuning measured for a TESLA cavity at different gradients.

\section{Microphonics}

Mechanical vibrations caused by the accelerator environment such as vacuum pumps and roots pump at the cryogenics facility are always present and may be transferred to accelerating structures through beam pipe, tuning frame and transfer lines. The frequency spectrum and amplitude of excitation depends strongly on the coupling to the cavities and the mechanical resonance frequencies and associated quality factor. Measurements at CEBAF and the Tesla Test Facility show typical excitation amplitudes of the order of $5-10 \mathrm{~Hz}(\mathrm{rms})$ and frequencies ranging from 0.1 $\mathrm{Hz}$ up to a few hundred $\mathrm{Hz}$ reflecting mechanical reso- 
nance frequencies and spectral components in the excitation spectrum.

\section{Beam Loading}

The loaded Q is usually chosen for matched conditions such that the all of the generator power (with the exception of a small amount dissipated in the sc-cavities) is transferred to the beam under design operating conditions. In the case of on-crest operation the magnitude of the beam induced voltage is half of the generator induced voltage. In the case of the TTF the relative transients induced by individual bunches are 1/700 which cannot and need not to be controlled by the RF controlled system if the droop is the same for all bunches. In the case of $\mathrm{H}$ - linacs (except SPL) the chopped nature of the beam leads to a drop within a bunch train which is of the order of $1 / 1000$ (SNS as example) and leads to a time varying energy gain which could be compensated only at the expense of twice the klystron power. The amplitude stability requirements permit however such a droop which is accepted. Slow bunch charge fluctuations within the bandwidth of the low level RF system can however be controlled.

\section{DESIGN CHOICES}

The basic idea of any RF control system is based on feedback control in which the cavity field vector is measured and compared to the desired set-point. The resulting error vector (=difference between measured field and set-point) is filtered and amplified before modulating the klystron drive and thereby the incident wave to the cavities. During pulsed operation, the perturbations from Lorentz force detuning and beam loading are of repetitive nature and the feedback can be supplemented by a feedforward which compensates the average repetitive error such that the feedback must correct only deviations from this pattern. Also the cavity field set point can be implemented as a table to accommodate the time-varying gradient and phase during cavity filling.

\section{Control Concepts}

The basic options for driving a cavity are the self-excited loop (SEL) and the generator driven resonator (GDR) which are shown in Figure 4. The conditions for operation of the SEL are a loop gain $>1$ and a loop phase shift of multiple of $360 \mathrm{deg}$. which can be set by the loop phase shifter. Therefore the SEL will operate at the cavity resonance frequency without the need for an external generator. A power limiter is necessary to protect cavity and coupler from excessive power. An amplitude feedback loop will maintain a stable gradient while phase lock with respect to an external reference can be achieved by use of the loop phase shifter. The GDR employs an external generator to drive the cavity while amplitude and phase are controlled using separate detector and controllers for amplitude and phase.
While the behavior of SEL and GDR is exactly the same during flat-top (or steady state in CW operation) the startup will be different. The start-up of the SEL is a statistical process and relies on noise power from the klystron. It is therefore not well defined and can be slow (several cavity time constants). The process can be accelerated by injection of a seed signal which also defines the initial phase during start-up. The major advantage of the SEL is that it will follow changes in resonator frequency immediately and can therefore be used to establish gradient in cavities which are detuned by many bandwidths from the operating frequency. This is especially useful if tuning of the cavity resonance frequency is required before phase lock can be obtained and the beam is turned on.

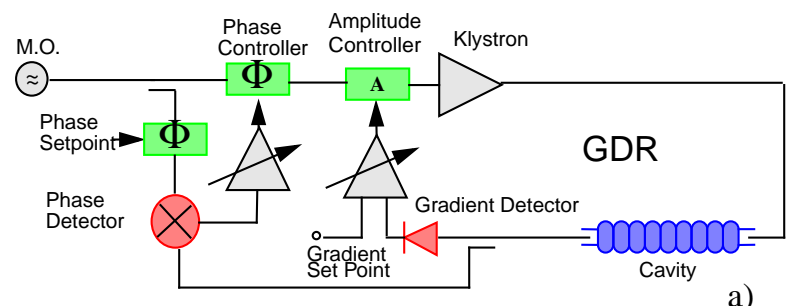

a)

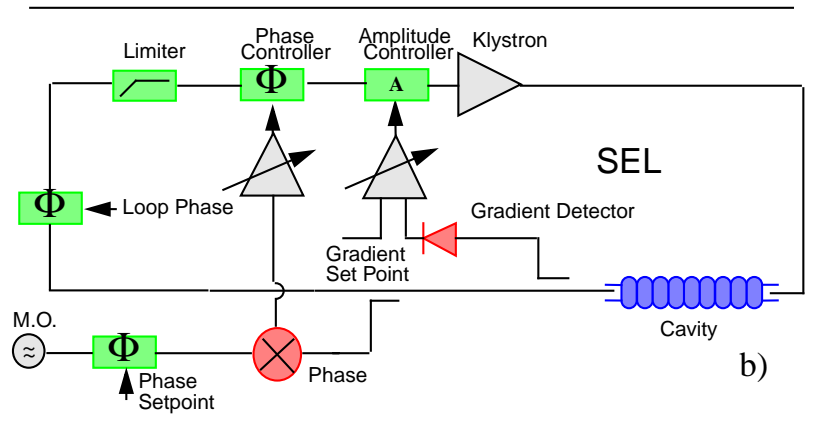

Fig. 3: a) Principle of generator driven resonator (GDR) and b) self-excited loop (SEL).

In pulsed operation where the cavity filling time should be as short a possible to maximize RF system efficiency, there will be not sufficient time to activate tuners after cavity filling before applying phase lock. Furthermore it is desirable to maintain a pre-determined filling curve for amplitude and phase to guarantee that the accelerating fields will be correct at the time of beam injection. This is best accomplished by a GDR system with a time-varying set point for the field vector during cavity filling. The GDR will ensure a well defined and controlled cavity filling.

\section{Control Algorithm}

The basic feedback mechanism is based on a proportional controller which can be supplemented by a integrator to further reduce the residual errors. In the pulsed operation the use of an integrator must be carefully evaluated since steady state might never be reached during the pulse and the integrator might introduce a slope on cavity gradient and phase. Since the dominating sources of perturbations such as beam loading and Lorentz force detuning are of 
repetitive nature, a feed forward system will already reduce most of the errors efficiently. Slow variations in RF system parameters dictate an adaptive feed forward system which corrects for these slow drifts.

\section{Other RF Control Issues}

The designer of a low level RF control system must decides whether to use Amplitude and Phase (A\&P) or Inphase and Quadratur-phase (I/Q) detectors and controllers and whether the system should be implemented with ana$\log$ or digital technology or as a hybrid system. While the I/Q concept allows for detection and control in all 4 quadrants including zero, is naturally better for control of cavity detuning $(\mathrm{Q}$ control already reduces the amplitude error) and is best for correction of large errors (best decoupling of control loops), the amplitude detector has the advantage of lower noise levels and can support control to the $10^{-4}$ level and is also more intuitive for operators who are used think in terms of amplitude and phase. It is also easier to design a power limiter for the drive signal of an A\&P controller than for an I/Q controller.

More issues that need to be considered by the LLRF designer are:

- Control of the cavity resonance frequency. Here the critical issues is the precise measurement of the cavity detuning which can be derived from the relationship of incident and reflected wave or especially attractive in the pulse case from the slope of the phase during decay of the cavity field following the RF pulse.

- Excitation of other passband modes by generator and beam. This is especially critical if harmonics of the beam coincide with other passband frequencies. Also field detectors may not detect the actual field seen by the beam.

- Aliasing effects by digital feedback system.

- Operation close to klystron saturation will result in strong dependency of loop gain with klystron output power.

- In case of vector-sum control the phase of the incident wave (and loaded Q) of each cavity must be controllable by means of remotely controlled wave guide tuners or phase shifters.

- Exception handling. In case of interlock trips or abnormal operating conditions (wrong loop phase or completely detuned cavity) the control system must ensure safe procedures to protect hardware and avoid unnecessary beam loss.

\section{RF CONTROL FOR THE TTF}

Fast amplitude and phase control is accomplished by modulation of the RF signal to the klystron which will drive up to 32 cavities [2]. While the TTF Linac employs a genera-

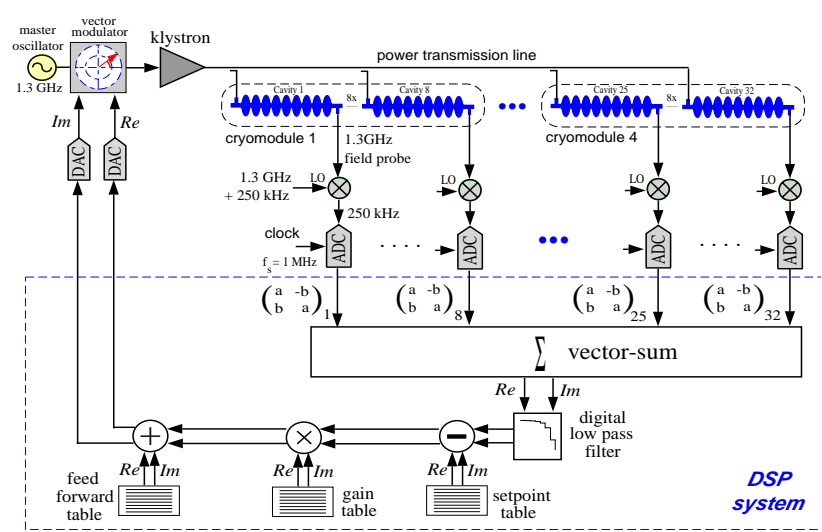

Fig. 4: Schematic of the digital RF control at the Tesla Test Facility

tor driven system, the booster cavity following the rf gun is operated in a self-excited loop [3].

\section{Design}

Digital I/Q detectors are used for the cavity field, and incident and reflected waves. The RF signals are converted to an intermediate frequency of $250 \mathrm{kHz}$ and sampled at a rate of $1 \mathrm{MHz}$ (i.e. two consecutive measurements describe I and $Q$ of the cavity field). The cavity field vector defined by I and Q is multiplied by $2 \times 2$ rotation matrices to correct for phase offsets and to calibrate the gradients of the individual cavity probe signals. The vector-sum is calculated and corrected for systematic measurements errors. Finally the set point is subtracted and the compensator filter is applied to calculate the new actuator setting (I and Q control inputs to the vector-modulator). Feedforward is added from a table in order to minimize the control effort. The feedforward tables are adaptively updated to reflect slow changing parameters such as average cavity detuning, changes in klystron gain, phase shift in the feedforward path, and general changes in operating parameters. The operation will be highly automated by the implementation of a finite state machine, which has access to high level applications including the adjustment of the loop phase, vector-sum calibration, frequency and waveguide tuner control, and exception handling.

\section{Performance}

Currently the TTF linac is operated with one klystron driving 16 cavities. The cavities have been routinely operated at a gradient of $15 \mathrm{MV} / \mathrm{m}$ providing a beam energy of 260 $\mathrm{MeV}$. The requirements of $\sigma_{A} / A<2 \cdot 10^{-3}$ for amplitude stability and $\sigma_{\phi}<0.5^{\circ}$ phase stability have been achieved with feedback only, the stability being verified by beam measurements. The residual fluctuations are dominated by a repetitive component which is further reduced by the adaptive feedforward by about one order of magnitude, thereby exceeding the design goals significantly. 


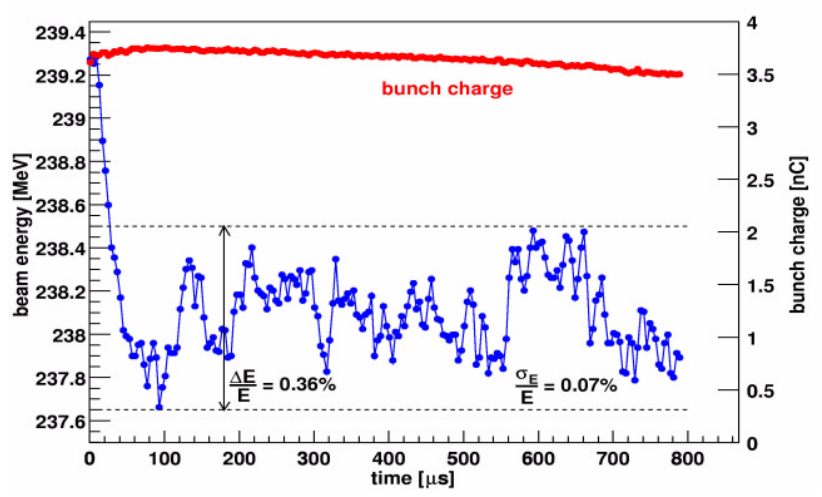

Fig. 5: RF System control performance with long beam pulses of $900 \mu \mathrm{s}$ at $8 \mathrm{~mA}$.

\section{RF CONTROL FOR H LINACS}

The RF control for non-relativistic beam is complicated by the fact that variations in the beam momentum lead to changes in arrival time at the individual cavities and therefore a change in momentum gain. Due to this phase slippage the energy spread at the end of the linac exhibits a larger sensitivity to cavity field fluctuations than in the case of relativistic particles. In the case of vector-sum control, parameter variations such as loaded Q or Lorentz force detuning constant $[4,5]$ can lead to excessive slopes on the individual cavity gradients. If the number of cavities in the vector-sum becomes large, the length of the associated section will become comparable to the synchrotron oscillation period lead to excessive differentials in beam loading and subsequently cavity fields of the involved cavities.

The RF control design for the SNS [6,7] employs a fully digital design with latest DSP and DPLD technology. The DPLD is applied in the feedback loop to minimize the time delay to about 1.1 us while the DSP will perform complex algorithms to implement a learning system for the feedforward control.

\section{DIAGNOSTICS}

Diagnostics are required for calibration of gradient and phase with respect to beam, loop phase, incident wave and reflected wave, cavity detuning, loaded Q, etc.

- Loop Phase. In case of the GDR the loop phase is determined during open loop operation by comparison of the vector controlling the actuator and the field vector induced in the cavity. In case of the SEL the loop phase is adjusted for maximum gradient (constant generator power) or minimum forward power (constant gradient, amplitude loop closed) to determine on resonance operation.

- Gradient and phase. Initial coarse calibration with RF, precise calibration with beam induced transients.
- Detuning and loaded Q. During decay of the cavity field the slope of gradient and phase (with respect to master oscillator) determine detuning and loaded Q.

\section{OUTLOOK}

Pulsed operation of superconducting cavities has been successfully demonstrated at the TTF and has proven that the phase and amplitude stability requirements even in the case of control of the vector-sum can be meet for the TESLA linear collider. Based on experience at the TTF, several $\mathrm{H}$ - linacs under construction or in planning have adopted pulsed superconducting cavities in their design, several even employing control of the vector-sum of a small number of cavities. Sensitivity to Lorentz force detuning and microphonics is reduced in these linac due to the high beam loading and the resulting low loaded Q factor, but the non-relativistic beams require tight field control to avoid excessive energy spread through phase slippage in the linac.

The future developments will be able to make use of advances in electronics. Faster DSPs and CPLDS reduce latency and allow more complex algorithms. Linearization of the klystron characteristics (amplitude and phase) will allow to operate the klystron very close to saturation while maintaining thigh field control. Phase and gradient calibration based on beam induced transient can be improved to detect small transients with high precision thereby reducing beam loss in the accelerator when phasing the linac. In the future one can envision global RF control systems which control beam momentum and in case of nonrelativistic beam the arrival time at the cavities instead of the cavity fields. This however requires precise and fast beam measurements which could be based on beam induced transients.

\section{REFERENCES}

[1] A. Mosnier, "Energy Stability in High Intensity Pulsed SC Proton Linac", LINAC 2000, Monterey, August 2000

[2] R. Brinkmann et al., "TESLA Technical Design Report, Part II", DESY 2001-011.

[3] A. Mosnier, S. Chel, B. Phung Ngoc, J.M. Tessier, "RF Control System for the SC cavity of the Tesla Test Facility Injector", PAC 97.

[4] N. Ouchi, E. Chishiro, E, T. Tsukishima, K. Mukugi, "Pulsed SC Proton Linac”, LINAC 2000, Monterey, August 2000.

[5] J. Tückmantel,"SPLinac', A Program to Simulate SC Linac RF Systems with Beam", CERN-SL-NOTE-2000-053 HRF

[6] A. Regan, "The SNS Linac RF Control System", this conference

[7] Y.M. Wang, S.I. Kwon, and A.H. Regan, "Analysis of the SNS Superconducting RF Control System”, LINAC 2000. 